\title{
Comparison of three suture-bridge techniques for large or massive rotator cuff tear with delamination
}

\author{
Atsushi Okubo ${ }^{1}$, Tadahiko Yotsumoto ${ }^{1, *}$, Nobuyoshi Watanabe ${ }^{1}$, Teruyoshi Kajikawa ${ }^{1}$, \\ Shun Nakajima ${ }^{1}$, Yasushi Oshima ${ }^{2}$, Norishige Iizawa ${ }^{2}$, and Tokifumi Majima ${ }^{2}$ \\ 1 Department of Orthopaedic Surgery, Kyoto Kujo Hospital, 10 Karahashi Rajomon, Minami-ku, Kyoto 601-8453, Japan \\ 2 Department of Orthopaedic Surgery, Nippon Medical School, 1-5-5 Sendagi, Bunkyo-ku, Tokyo 113-8602, Japan
}

Received 5 April 2021, Accepted 4 July 2021, Published online 16 August 2021

\begin{abstract}
Introduction: Rotator cuff tear with delamination is considered a risk factor for postoperative retear. The purpose of this study was to compare clinical outcomes between three repair procedures for large or massive rotator cuff tears with delamination: conventional en masse suture bridge (EMSB), double-layer suture bridge (DLSB), and the combination of DLSB with modified Debyere-Patte (DLSB + DP). Methods: 53 shoulders of 52 patients who had massive rotator cuff tears with delamination were categorized into three groups: EMSB (18 shoulders), DLSB (24 shoulders), and DLSB + DP (11 shoulders). The mean postoperative follow-up period was 34.6 months. Pre- and postoperative evaluations included a range of motion (ROM), Constant scores, global fatty degeneration (GFDI), and tendon integrity according to Sugaya's classification by magnetic resonance images (MRI). Results: In all groups, ROM significantly improved after the procedures. Mean constant scores significantly improved: from 45.5 to 77.4 after EMSB, from 45.5 to 87.6 after DLSB, and from 46.3 to 88.0 after DLSB + DP. Significant differences were noted in postoperative Constant scores ( $p=0.018$ : DLSB vs. EMSB, and $p=0.045$ : DLSB + DP vs. EMSB). The Constant pain scores were better for DLSB + DP than for EMSB $(p=0.012)$. Global fatty degeneration index (GFDI) with DLSB + DP was significantly higher than that for either EMSB or DLSB, indicating significant preoperative fatty degeneration for DLSB + DP. Retear occurred in $27.8 \%$ of the EMSB group, $12.5 \%$ of the DLSB group, and $9.1 \%$ of the DLSB + DP group. Discussion: Comparisons of the three groups demonstrated that DLSB and DLSB + DP achieved better clinical outcomes than EMSB for the repair of large or massive rotator cuff tears. DLSB + DP is useful for massive rotator cuff tears with severe fatty degeneration or for cases where the presence of excessive tension is anticipated when repairing the torn cuff.
\end{abstract}

Key words: Rotator cuff tear, Delamination, Suture-bridge, En masse repair, Double-layer repair.

\section{Introduction}

The delamination of the rotator cuff has been described as a horizontal lesion between the superficial and deep layers [1, 2]. Delamination is observed in $36-82 \%$ of rotator cuff tear cases, and its presence is considered a risk factor for postoperative retear after rotator cuff repair [3-7]. An optimal fixation method for a rotator cuff stump with delamination has yet to be established.

Double-row double-layer fixation is reported as a repair method of the stump of delaminated rotator cuff tears. However, there is a high incidence of retear in large and massive rotator cuff tear cases [2]. The suture bridge (SB) is a tendon stump fixation method for rotator cuff tears with a strong initial fixing force and provides favorable cuff integrity with its broad coverage footprint. The en masse suture bridge (EMSB) has

\footnotetext{
*Corresponding author: sssyotsu@yahoo.co.jp
}

been used for delaminated rotator cuff tears, with an experimental study of the method demonstrating successful histological and biomechanical characteristics of cuff healing [8]. Mochizuki et al. noted the effectiveness of the double-layer suture bridge (DLSB) technique, which independently repairs the superficial layer (infraspinatus) and deep layer (articular capsule). However, they did not describe the clinical results [9]. The rotator cuff's tear size and fatty degeneration are reported risk factors for retear after arthroscopic rotator cuff repair (ARCR) [10]. Several other repair methods of large or massive tears, such as musculotendinous advancement, have also been reported, including a modified Debeyre-Patte (DP) procedure reported effective for large or massive tears unable to be covered to the footprint by torn stumps even though sufficiently mobilized [11-13].

To our knowledge, there have been no comparative studies including muscular advancement-combined DLSB for patients with degenerative delaminated rotator cuff tears. Starting in 
Table 1. Patient demographics.

\begin{tabular}{lcccc}
\hline & EMSB $(n=18)$ & DLSB $(n=24)$ & DLSB + DP $(n=11)$ & $4 / 7$ \\
\hline Sex, $n$ (males/females) & $12 / 6$ & $11 / 12$ & $7 / 4$ & .267 \\
Dominant/non-dominant side & $12 / 6$ & $19 / 5$ & $72.9 \pm 6.1$ & .568 \\
Age (years) & $69.0 \pm 6.1$ & $69.6 \pm 9.5$ & $26.7 \pm 4.7$ & .483 \\
Follow-up period (months) & $31.5 \pm 14.0$ & $34.9 \pm 11.7$ & .061 \\
\hline
\end{tabular}

Notes: Data are presented as the means $\pm S D$.

2010, we began using EMSB when treating delamination of the rotator cuff; however, some cases showed poor prognosis. To improve clinical results, we gradually shifted the surgical method to the double layer suture bridge method (DLSB). The purpose of our study was to retrospectively compare clinical outcomes after arthroscopic repair of delaminated rotator cuff tears which employed DLSB, EMSB, or DLSB + DP methods; and then consider an optimum repair method for the specific pathological condition.

\section{Materials and methods}

Among patients surgically treated by arthroscopic rotator cuff repair (ARCR) at our hospital, between September 2011 and June 2018, there were 53 shoulders (52 patients) presenting massive rotator cuff tears, according to the DeOrio and Cofield classification, with delamination. These 52 patients were selected as subjects for the study. Inclusion criteria were a large or massive rotator cuff tear undergoing ARCR, and a minimum follow-up of 2 years. Exclusion criteria were patients undergoing revision rotator cuff repair, or clinical findings of instability.

The subjects were then classified into three groups based on the treatment method. The EMSB group: superficial and deep layers fixed by en bloc suturing using suture anchors. The DLSB group: superficial and deep layers individually fixed by sutures reported by Mochizuki et al. [9]. The DLSB + DP group: a modified DP procedure described [13] was added after DLSB when the rotator cuff was unable to be covered up to the footprint though sufficiently mobilized.

As shown in Table 1, the EMSB group comprised 18 shoulders of 18 patients (12 males and 6 females, mean age of 69 years (range: 58-78 years)); the DLSB group comprised 24 shoulders of 23 patients (11 males and 12 females, mean age of 69.6 years (range: $49-87$ years)); and the DLSB + DP group comprised 11 shoulders of 11 patients (4 males and 7 females, at a mean age of 72.9 years (range: 67-87 years)). The mean postoperative follow-up observation durations were 31.5 months (range: 24-67 months) for the EMSB group; 34.9 months (range: $24-72$ months) for the DLSB group; and 26.7 months (range: 24-36 months) for the DLSB + DP group.

An institutional review board approved the study, and the enrolled subjects provided written informed consent for participation in the study.

\section{Surgical procedure}

ARCR was performed in the beach chair position under general anesthesia on all subjects. For retention of the upper limb, an arm holder (TRIMANO Arm Holder, Arthrex, Naples, FL, USA) was used. Five portals (posterior, anterior, anterolateral, posterolateral, and upper anchor) were used for the operation. For cases of subacromial decompression, subacromial synovectomy and subacromial osteophyte resection were performed. Prior to repair, the rotator cuff was sufficiently mobilized.

For the rotator cuff repair method, 1-2 medial anchors and 2-3 lateral anchors were set corresponding to the tear size using suture anchors (HEALIX, DePuy Synethes Mitek Sports Medicine, Raynham, MA, USA). For EMSB, the superficial and deep layers were fixed en bloc using a medial anchor thread (Figure 1). For DLSB, first, the medial anchor thread was passed through the deep layer to fix and suture by adding sliding knots and two half-hitches, as described by Mochizuki et al. [9]; then, all threads were passed through the superficial layer without cutting the anchor thread and fixed using the lateral anchor (Figure 1). In cases unable to be covered up to the footprint despite the rotator cuff being sufficiently mobilized, an approximately $3 \mathrm{~cm}$ transverse skin incision was made outward from the medial border of the spine of the scapula following the method reported by Morihara et al. [13]. This modified DP procedure dissecting the supraspinatus and infraspinatus muscles was done while maintaining continuity to the fascia of the rhomboideus muscle (Figure 2). Then, suture fixation of the stump was performed by DLSB.

\section{Rehabilitation}

In the EMSB and DLSB groups, a postoperative shoulder abduction brace was attached for 3-4 weeks, corresponding to the tear size. In the DLSB + DP group, the abduction brace was attached for 6 weeks following surgery. Passive range of motion (ROM) training was initiated on the day after surgery, and active ROM training was initiated upon removal of the abduction brace. Resistance exercise was initiated 12 weeks after surgery.

\section{Clinical outcomes}

Clinical outcome assessments included pre- and postoperative ROM (flexion, abduction, external rotation, internal rotation), Constant scores, and the global fatty degeneration index (GFDI). The latter, representing the preoperative degree of fatty infiltration into the rotator cuff as observed on MRI, was defined as the mean for the supraspinatus, infraspinatus, and subscapularis muscles, according to the Goutallier classification [14, 15]. Postoperative cuff integrity was evaluated using Sugaya's classification, with Types IV and V regarded as retear [16]. 


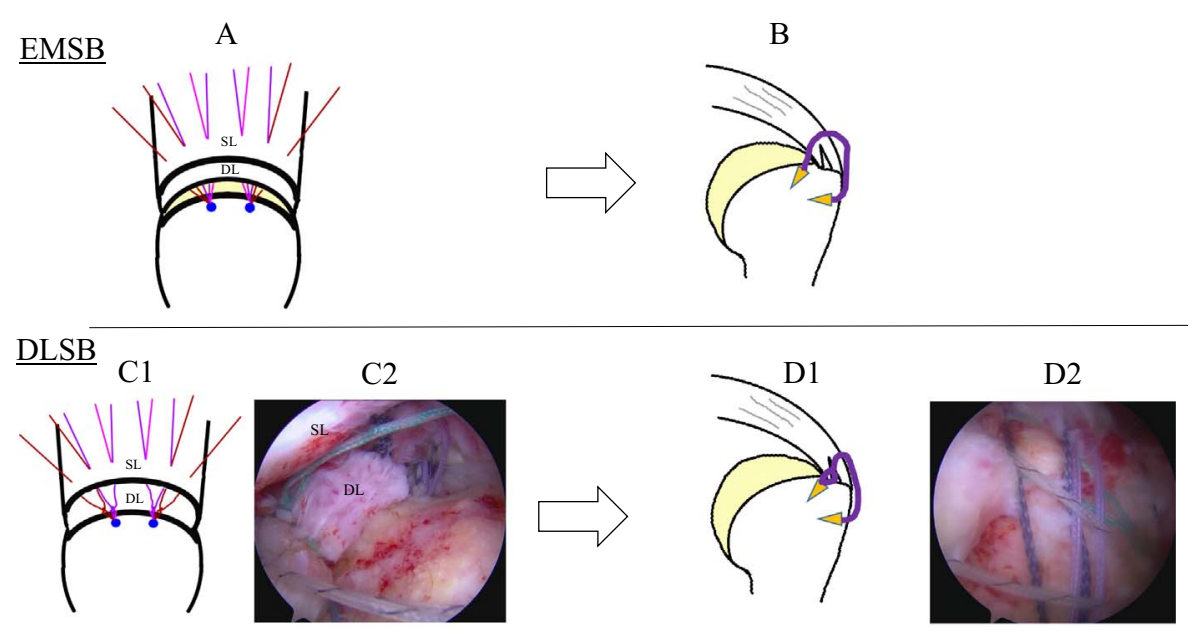

Figure 1. EMSB and DLSB procedures. For EMSB, a suture is passed through the superficial and deep layers (A) and bridged with lateral anchors (B). For DLSB, the superficial layer is first repaired with medial anchors (C); then, the remaining sutures are passed through the deep layer and bridged with lateral anchors (D). Abbreviations: EMSB: en masse suture bridge, DLSB: double layer suture bridge, SL: superficial layer, DL: deep layer, C2 and D2: arthroscopic images of the right shoulder as viewed from the posterolateral portal.

A. Preoperative cross-sectional view

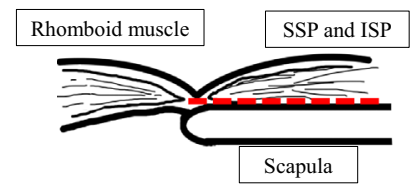

B1. Intra-operative posterior view

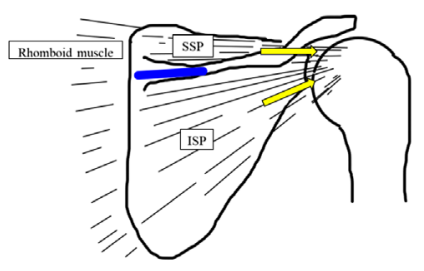

B2. Intra-operative cross-sectional view

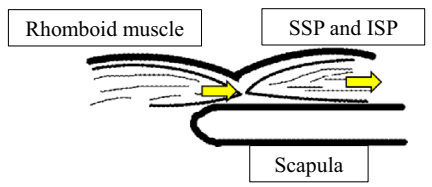

Figure 2. Schema of modified Debyere-Patte (DP) technique. (A) Preoperative cross-sectional view. (B1) Intra-operative posterior view of the right shoulder; (B2) Intra-operative cross-sectional view. Red dotted line: SSP and ISP, while maintaining continuity with the rhomboid fascia, were detached from the medial edge of scapular and advanced laterally. Blue line: skin incision. Yellow arrow: pulling direction of torn tendons with the rhomboids. Abbreviations: SSP: supraspinatus muscle, ISP: infraspinatus muscle. (Modified illustration of Morihara et al. [13]).

\section{Statistical analysis}

Statistical analysis was done using EZR (Ver. 1.50, Saitama Medical Center, Jichii Medical University, Saitama, Japan). For all three groups, pre- and postoperative ROM and Constant scores were analyzed using the Mann-Whitney $U$-test; KruskalWallis was used for pre- and postoperative comparisons, and Fisher's test was used for comparison of the incidence of retear, and a $p$-value of $<0.05$ was considered statistically significant.

\section{Results}

\section{Range of motion}

Each surgical group showed significant postoperative active ROM improvement, including flexion, external rotation, and internal rotation degrees (Table 2). In the EMSB group, mean ROM before the operation was flexion (Flex) $105.6^{\circ}$, external rotation (ER) $49.7^{\circ}$, internal rotation (IR) L 4.8; after the last follow-up, it was Flex $158.9^{\circ}$, ER $67.2^{\circ}$, and IR L 2, respectively. In the DLSB group, ROM improved from Flex $109^{\circ}$, ER $47.2^{\circ}$, IR L 4.5 to Flex $159.6^{\circ}$, ER $71.4^{\circ}$, and IR L 2, respectively. In the DLSB + DP group, ROM improved from Flex $108.2^{\circ}$, ER $43.2^{\circ}$, IR L 3.6 to Flex $149.1^{\circ}$, ER $69.1^{\circ}$, and IR L 1.3, respectively. No significant differences were noted in either pre- or postoperative ROM improvement among the three groups (Table 2).

\section{Constant scores}

The mean Constant scores for the EMSB, DLSB, and DLSB + DP groups, respectively, were $45.5 \pm 14.3$, 
Table 2. Active range of motion before and after treatment.

\begin{tabular}{|c|c|c|c|c|}
\hline & EMSB & DLSB & $\mathrm{DLSB}+\mathrm{DP}$ & $P$-values \\
\hline \multicolumn{5}{|l|}{ Flex } \\
\hline Pre-operation & $105.6 \pm 50.0$ & $109 \pm 30.1$ & $108.2 \pm 43.2$ & .979 \\
\hline Last follow-up & $158.9 \pm 20.5$ & $159.6 \pm 19.7$ & $149.1 \pm 26.4$ & .509 \\
\hline \multicolumn{5}{|l|}{ ER } \\
\hline Pre-operation & $49.7 \pm 16.8$ & $47.2 \pm 20.0$ & $43.2 \pm 25.6$ & .623 \\
\hline Last follow-up & $67.2 \pm 13.7$ & $71.4 \pm 14.9$ & $69.1 \pm 10.8$ & .4 \\
\hline \multicolumn{5}{|l|}{ IR } \\
\hline Pre-operation & L 4.8 & L 4.5 & L 3.6 & .27 \\
\hline Last follow-up & L 2 & L 2 & L 1.3 & .502 \\
\hline
\end{tabular}

Note: Data are presented as the means $\pm S D$. Abbreviations: Flex: degree of flexion; ER: degree of external rotation; IR: internal rotation; L: Lumbar spine.

$45.5 \pm 11.6$, and $46.3 \pm 11.2$ before surgery, and $77.4 \pm 13.6$, $87.6 \pm 11.4$, and $88.0 \pm 10.5$ after surgery achieving favorable improvement in all groups (Figure 3A). Significant differences were noted in postoperative Constant scores between the EMSB and DLSB groups $(p=0.018)$ and between the EMSB and DLSB + DP groups $(p=0.045)$. In addition, a significant difference was noted in the postoperative pain of the Constant score between the EMSB and DLSB + DP groups $(p=0.012)$ (Figure 3B). No significant differences were noted in postoperative ADL and strength of the constant scores among the three groups (Figures 3C and 3D).

\section{Global fatty degeneration index}

The mean preoperative GFDI was $1.52 \pm 0.4,1.80 \pm 0.5$, and $2.28 \pm 0.4$, respectively, for the EMSB, DLSB, and DLSB + DP groups, with the latter group significantly higher than the other two, and indicating significant preoperative fatty degeneration ( $p=0.015$ : DLSB vs. DLSB + DP, and <0.001: DLSB + DP vs. EMSB) (Table 3).

\section{Postoperative cuff integrity}

Postoperative retear occurred in 5 (27.8\%), 4 (16.0\%), and 1 subject $(9.09 \%)$, respectively, of the EMSB, DLSB, and DLSB + DP groups, reflecting the low incidence of retear in the DLSB + DP group. No statistically significant differences among the groups were found (Table 3), and no other postoperative complications were noted.

\section{Discussion}

Large and massive rotator cuff tears with delamination are problematic due to the risk of retear because of the anatomical characteristics of the horizontal superficial and deep layers [1-7]. Various surgical repair procedures have been used though an optimal surgical procedure has yet to be determined $[2,8,9]$. In this study, three surgical procedures, EMSB, DLSB, and DLSB + DP, were compared and investigated for their clinical outcomes treating massive rotator cuff tear. The retear rate was the lowest for the DLSB + DP group, even though its mean preoperative GFDI was the highest.
There were limitations to our study in that it was a retrospective investigation, there was a small sample size, and a lack of a priori sample size calculation. In addition, the timing for the surgical choices of EMSB and DLSB may have influenced clinical outcomes. However, to our knowledge, no reported studies are comparing the EMSB and DLSB methods with the muscular advancement-combined DLSB method for patients with massive degenerative delaminated rotator cuff tears.

The suture bridge method reportedly provides excellent rigidity compared with the single-row or double-row methods because of greater contact area and contact pressure [2, 17-19] and has been frequently used for rotator cuff tear. However, the incidence of reported retear remains high in cases of massive rotator cuff tear with severe fatty degeneration of the rotator cuff [15], and an optimum treatment method remains controversial though several treatment methods were reported for irreparable rotator cuff tear by primary repairs, such as partial repair, patch, musculotendon transfer, muscular advancement, and superior capsular reconstruction.

Delamination is often observed, which tends to show tearing in the posterior region [20]. From a histological standpoint, there has been no consistent agreement on the developmental mechanism of the two-layered delamination structure [5]. The rotator cuff is comprised of five histologically different layers, and delamination tends to develop between the 2nd and 3rd layers with different fiber distributions [21, 22]. Delamination is a risk factor for postoperative retear after rotator cuff repair, thus special attention to its presence and extent is considered important [3-5]. Delamination repair methods have included suturing the superficial and deep layers en bloc [23], individually suturing superficial and deep layers, and suturing only the superficial layer [9, 24, 25]. Mochizuki et al. reported that the superficial and deep layers should be individually sutured as their footprints are anatomically different [9]. A study describing the usefulness of DLSB, as compared to EMSB, concluded the advantage came from repairing the superficial and deep layers to an anatomically appropriate footprint [25]. Cha et al. stated risk of retear in cases with severe rotator cuff degeneration even when the dual-layer method was used, suggesting the need for an alternative suture method and additional treatment for severe degeneration of the rotator cuff [24].

For the subjects of this study, DLSB was done in combi nation with the modified DP method for cases considered 
A

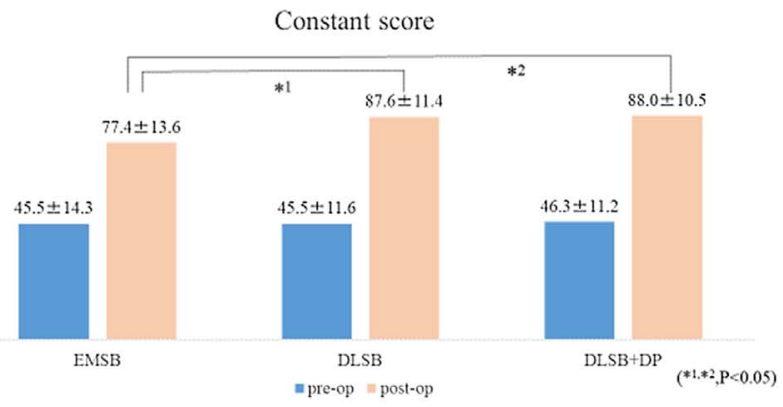

$*$ p-values $=0.986$ (DLSB vs. DLSB+DP), $0.018^{* 1}$ (DLSB vs. EMSB) , and $0.045^{* 2}$ (DLSB+DP vs. EMSB)

$\mathrm{C}$

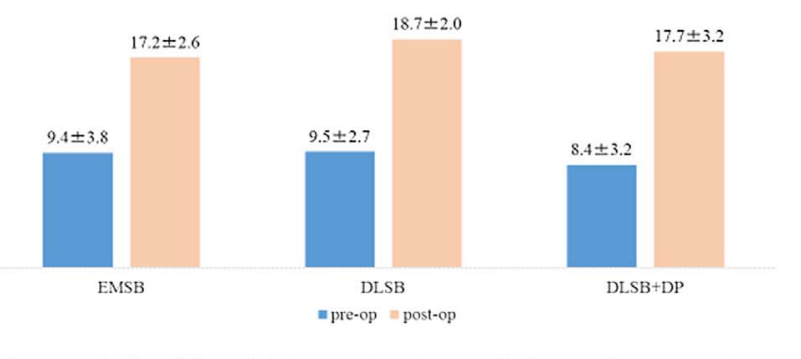

$\mathrm{B}$

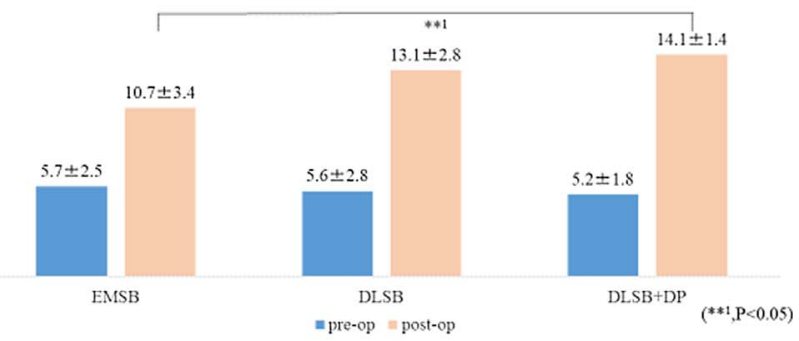

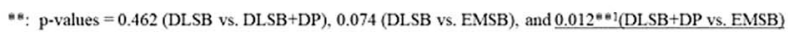

$\mathrm{D}$

Constant score:Strength

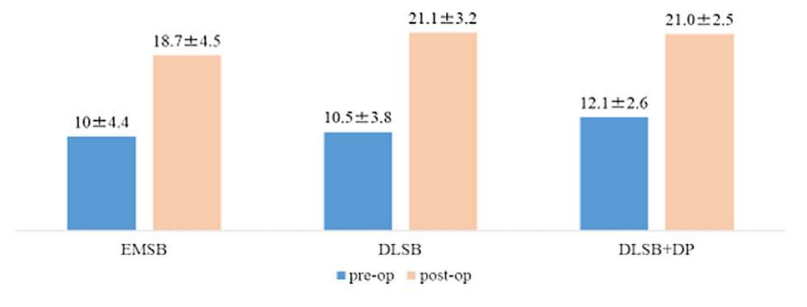

Figure 3. Clinical outcome assessment: Constant Scores. (A) Significant improvements were noted in the postoperative Constant scores as between the EMSB and DLSB groups $(p=0.018)$, and between the EMSB and DLSB + DP groups $(p=0.045)$. (B) Postoperative pain Constant score: a significant difference was noted in the postoperative pain between the EMSB and DLSB + DP groups $(p=0.012)$. $(\mathrm{C})$ Postoperative ADL. (D) Strength of the constant scores. No significant differences were noted in the postoperative ADL and strength of the Constant scores among the three groups.

Table 3. Radiological findings on MRI.

\begin{tabular}{lcccc}
\hline & EMSB $(n=18)$ & DLSB $(n=24)$ & DLSB + DP $(n=11)$ & $P$-values \\
\hline Preoperative GFDI & $1.52 \pm 0.4^{1}$ & $1.80 \pm 0.5^{2}$ & $2.28 \pm 0.4^{1,2}$ & $\leq .001^{*}$ \\
Postoperative retear, $n(\%)$ & $5(27.8 \%)$ & $3(12.5 \%)$ & $1(9.1 \%)$ & .395 \\
\hline
\end{tabular}

Notes: Data are presented as the means $\pm S D$. Abbreviation: GFDI: global fatty degeneration index.

${ }^{*} p<0.05$.

${ }_{1}^{1} p=0.015$ (DLSB vs. DLSB + DP), 0.107 (DLSB vs. EMSB),

${ }^{2} p<0.001$ (DLSB + DP vs. EMSB). GFDI in the DLSB + DP group was significantly higher than that of both EMSB and DLSB groups.

irreparable by the primary treatment. No significant differences were noted in postoperative ROM among the EMSB, DLSB, and DLSB + DP groups. Still, the postoperative Constant scores for the EMSB group were significantly inferior to those for the DLSB and DLSB + DP groups. The outcome of pain of the Constant scores in the EMSB group, which had the highest incidence of retear, was significantly inferior to that in the DLSB + DP group, which had the lowest incidence of retear. Statistically, the incidence of retear was not significantly different between the three groups. However, in the DLSB + DP group, the retear ratio was the lowest and the preoperative GFDI the highest and significantly higher than that for DLSB. This suggests the efficacy of the modified DP method in reducing the tension of the repaired rotator cuff and possibly of DLSB + DP preventing retear in cases with severe degeneration of the rotator cuff. To further verify our findings, a long-term outcome evaluation is considered necessary.

\section{Conclusion}

DLSB method is considered a better procedure for the successful anatomical repair of a delaminated rotator cuff tear of the shoulder. Further, the DLSB + DP method may be a useful surgical procedure for patients with a large-sized tear, severe fatty degeneration of the rotator cuff, or when excessive tension is present when repairing the rotator cuff.

\section{Conflict of interest}

The authors declare that they have no conflicts of interest in relation to this article.

\section{Funding}

The authors received no special funding related to this study. 


\section{Ethical approval}

The institutional review board of Kujo Hospital, Japan, approved this study.

\section{Informed consent}

All subjects were provided explanations and signed a written consent form regarding this study.

\section{Authors' contributions}

A. Okubo: Designed the study, performed operations, acquired data, and analyzed results. T. Yotsumoto: Provided clinical advice and critically revised the contents of the manuscript. N. Watanabe, T. Kajikawa, and Shun Nakajima: Participated in the clinical treatment of patients and the improvement of this manuscript. Y. Oshima and N. Iizawa: Made critical comments on the contents of the manuscript and assisted in its completion. T. Majima: Comprehensively reviewed and gave suggestions to improve this manuscript.

\section{References}

1. Minagawa H, Itoi E, Konno N, Kido T, Sano A, Urayama M, et al. (1998) Humeral attachment of the supraspinatus and infraspinatus tendons: An anatomic study. Arthroscopy 14(3), 302-306.

2. Sugaya H, Maeda K, Matsuki K, Moriishi J (2005) Functional and structural outcome after arthroscopic full-thickness rotator cuff repair: Single-row versus dual-row fixation. Arthroscopy 21(11), 1307-1316.

3. Boileau P, Andreani O, Schramm M, Baba M, Barret H, Chelli M (2019) The effect of tendon delamination on rotator cuff healing. Am J Sports Med 47(5), 1074-1081.

4. Boileau P, Brassart N, Watkinson DJ, Carles M, Hatzidakis AM, Krishnan SG (2005) Arthroscopic repair of full-thickness tears of the supraspinatus: Does the tendon really heal? J Bone Joint Surg Am 87(6), 1229-1240.

5. Han Y, Shin JH, Seok CW, Lee CH, Kim SH (2013) Is posterior delamination in arthroscopic rotator cuff repair hidden to the posterior viewing portal? Arthroscopy 29(11), 1740-1747.

6. Iwashita S, Hashiguchi H, Okubo A, Yoneda M, Takai S (2018) Characteristics of the patients with delaminated rotator cuff tear. SICOT-J 4, 28.

7. MacDougal GA, Todhunter CR (2010) Delamination tearing of the rotator cuff: Prospective analysis of the influence of delamination tearing on the outcome of arthroscopically assisted mini open rotator cuff repair. J Shoulder Elbow Surg 19(7), 1063-1069.

8. Cheon SJ, Kim JH, Gwak HC, Kim CW, Kim JK, Park JH (2017) Comparison of histologic healing and biomechanical characteristics between repair techniques for a delaminated rotator cuff tear in rabbits. J Shoulder Elb Surg 26(5), 838-845.

9. Mochizuki T, Nimura AMiyamoto T, Koga H, Akita K, Muneta T (2016) Repair of rotator cuff tear with delamination: Independent repairs of the infraspinatus and articular capsule. Arthrosc Tech 5 (5), e1129-1134.

10. Kim KC, Shin HD, Lee WY (2012) Repair integrity and functional outcomes after arthroscopic suture-bridge rotator cuff repair. J Bone Joint Surg Am 94(8), e48.

11. Gerber C (1992) Latissimus dorsi transfer for the treatment of irreparable tears of the rotator cuff. Clin Orthop Relat Res (275), 152-160.

12. McLaughlin HL (1994) Lesions of the musculotendinous cuff of the shoulder. The exposure and treatment of tears with retraction. Clin Orthop Relat Res (304), 3-9

13. Morihara T, Kida Y, Furukawa R, Sukenari T, Kabuto Y, Kurokawa M, et al. (2018) Therapeutic outcomes of muscular advancement by an arthroscopic-assisted modified DebeyrePatte procedure for irreparable large and massive rotator cuff tears. J Orthop Sci 23(3), 495-503.

14. Goutallier D, Postel JM, Bernageau J, Lavau L, Voisin MC (1994) Fatty muscle degeneration in cuff ruptures: Pre- and postoperative evaluation by CT scan. Clin Orthop Relat Res (304), 78-83.

15. Goutallier D, Postel JM, Gleyze P, Leguilloux P, Van Driessche $S$ (2003) Influence of cuff muscle fatty degeneration on anatomic and functional outcomes after simple suture of fullthickness tears. J Shoulder Elb Surg 12(6), 550-554.

16. Sonnabend DH, Yu Y, Howlett CR, Harper GD, Walsh WR (2001) Laminated tears of the human rotator cuff: A histologic and immunochemical study. J Shoulder Elb Surg 10(2), 109-115.

17. Chen Y, Li H, Qiao Y, Ge Y, Li Y, Hua Y, et al. (2019) Doublerow rotator cuff repairs lead to more intensive pain during the early postoperative period but have a lower risk of residual pain than single-row repairs. Knee Surgery, Sport Traumatol Arthrosc 27(10), 3180-3187.

18. Mazzocca AD, Millett PJ, Guanche CA, Santangelo SA, Arciero RA (2005) Arthroscopic single-row versus double-row suture anchor rotator cuff repair. Am J Sports Med 33(12), 1861-1868.

19. Wall LB, Keener JD, Brophy RH (2009) Clinical outcomes of double-row versus single-row rotator cuff repairs. Arthroscopy 25(11), 1312-1318.

20. Sonnabend DH, Watson EM (2002) Structural factors affecting the outcome of rotator cuff repair. J Shoulder Elb Surg 11(3), 212-218.

21. Clark JM, Harryman DT (1992) Tendons, ligaments, and capsule of the rotator cuff. Gross and microscopic anatomy. J Bone Joint Surg Am 74(5), 713-725.

22. Keisuke M, Ryo M, Nobuyasu O, Surgery O (2006) Histological assessment of delamination observed in rotator cuff tears. Katakansetsu 30(3), 461-464.

23. Gwak HC, Kim CW, Kim JH, Choo HJ, Sagong SY, Shin J (2015) Delaminated rotator cuff tear: Extension of delamination and cuff integrity after arthroscopic rotator cuff repair. J Shoulder Elb Surg 24(5), 719-726.

24. Cha SW, Lee CK, Sugaya H, Kim TLee SC (2016) Retraction pattern of delaminated rotator cuff tears: Dual-layer rotator cuff repair. J Orthop Surg Res 11(1), 75.

25. Nakamizo H, Horie R (2018) Comparison of en masse versus dual-layer suture bridge procedures for delaminated rotator cuff tears. Arthroscopy 34(12), 3150-3156. 